\title{
Three Proofs that the Square Root of 2 Is Irrational
}

\author{
Shalin Shah \\ sshah100@jhu.edu \\ shah.shalin@gmail.com
}

This short article surveys three proofs that $\sqrt{2}$ is irrational. The first proof is a simple proof by contradiction and the second and third proofs use field theory from abstract algebra.

Keywords: $\sqrt{2}$, irrational numbers, rational numbers, field theory, Galois automorphism, square root of 2, Eisenstein criterion, modern algebra

\section{Proof By Contradiction that $\sqrt{2}$ is irrational}

Lemma 1.1: $\sqrt{2}$ is irrational

Proof:

Assume that $\sqrt{2}$ is rational.

Then, let $\sqrt{2}=\frac{p}{q}$ where $p, q \in \mathbf{Z}$

Also assume that $p$ and $q$ are relatively prime i.e $\operatorname{gcd}(p, q)=1$

Then, $2=\frac{p^{2}}{q^{2}}$

$\Longrightarrow p^{2}=2 q^{2}$

$\Longrightarrow p$ is even, say $2 m$

$\Longrightarrow 4 m^{2}=2 q^{2}$

$\Longrightarrow 2 m^{2}=q^{2}$ 
$\Longrightarrow q$ is even.

Thus, both $p$ and $q$ are even and have 2 as a common factor.

But we assumed that $p$ and $q$ are relatively prime. This is a contradiction. Thus, $\sqrt{2}$ cannot be written as $\frac{p}{q}$ for $p, q \in \mathbf{Z}$

Thus $\sqrt{2}$ is irrational.

\section{Background on Fields}

\subsection{Groups}

A group $G$ is an algebraic structure with some properties. It is a set with a binary operation $*$ which satisfies the group axioms:

1. Closure: $\forall a, b \in G, a * b \in G$

2. Identity: $\forall a \in G$, there is an element $i$ such that $a * i=a$. The element $i$ is called the identity. For addition it is usually 0 and for multiplication it is usually 1 .

3. Inverses: $\forall a \in G$, there exists an element $a^{-1}$ such that $a * a^{-1}=i$ where $i$ is the group identity.

4. Associativity: $\forall a, b, c \in G,(a * b) * c=a *(b * c)$ i.e. it does not matter if $a$ and $b$ are multiplied first or $b$ and $c$ are multiplied first. For three elements, it is sufficient to write $a * b * c$ and leave out the brackets.

A group is called abelian or commutative if $a * b=b * a$.

\section{$2.2 \quad$ Fields}

A field is an algebraic structure, a set, which has the properties of two abelian groups with a distributive property.

A field $\mathbf{F}$ is a set consisting of an abelian group under addition and an abelian group under multiplication which obeys the distributive property:

$\forall a, b, c \in \mathbf{F}, a *(b+c)=a * b+a * c$

$\forall a, b, c \in \mathbf{F},(b+c) * a=b * a+c * a$

Thus, a field $\mathbf{F}$ has closure under addition and multiplication, it has an additive and multiplicative identities, it has additive and multiplicative inverses, 
both multiplication and addition are associative and multiplication distributes under addition.

\subsection{Field Automorphisms}

An automorphism on a set is a function from the set to itself. It is a bijection. An automorphism is also a homomorphism which preserves the field structure under both, addition and multiplication.

Let $\mathbf{F}$ be a field. A function $\phi: \mathbf{F} \rightarrow \mathbf{F}$ is an automorphism if it is a bijection and a homomorphism for both addition and multiplication.

$\forall a, b \in \mathbf{F}, \phi(a+b)=\phi(a)+\phi(b)$ and $\phi(a * b)=\phi(a) * \phi(b)$

\subsection{Extension Fields}

The rational numbers $\mathbf{Q}$ are a field. But the roots of the polynomial $x^{2}-2=0$ do not exist in $\mathbf{Q}$. The rationals can be extended by adding $\sqrt{2}$ to the field to create an extension field $\mathbf{Q}(\sqrt{2})$. Automorphisms can be defined on this extension field.

Let $\mathbf{F}=\mathbf{Q}(\sqrt{2})$ be the extension field.

Let $\phi: \mathbf{F} \rightarrow \mathbf{F}$ be an automorphism.

There are only a few possible automorphisms for $\phi$. One of them is:

$\forall a, b \in \mathbf{Q}, \phi(a+b \sqrt{2})=a-b \sqrt{2}$

It can be shown that the following are all possible Galois automorphisms:

1. $\forall a, b \in \mathbf{Q}, \phi(a+b \sqrt{2})=a+b \sqrt{2}$

2. $\forall a, b \in \mathbf{Q}, \phi(a+b \sqrt{2})=a-b \sqrt{2}$

The Galois group of an extension field is the group of all automorphisms.

\subsection{Two Lemmas}

Lemma 1.2: If $\phi$ is an automorphism $\phi: \mathbf{F} \rightarrow \mathbf{F}$ on an extension field of the rational numbers $\mathbf{Q}(\sqrt{2})$, then the automorphism sends $\mathbf{Z}$ to $\mathbf{Z}$.

Proof:

Let $\phi(1)=x$.

Clearly, $x \neq 0$ because otherwise, 
$\phi(2)=\phi(1+1)=\phi(1)+\phi(1)=0+0=0$ and $\phi$ would not be a bijection.

Now, $(\phi(1))^{2}=\phi(1) * \phi(1)=\phi(1 * 1)=\phi(1)$

Similarly, $(\phi(1))^{3}=\phi(1) * \phi(1) * \phi(1)=\phi(1 * 1 * 1)=\phi(1)$

Thus, $\phi(1)=1$

Now consider any $n \in \mathbf{N}$

$\phi(n)=\phi(1+1+1 \ldots n)=\phi(1)+\phi(1) \ldots=n$

Now, a homomorphism sends identity to identity.

Thus $\phi(0)=0$

Now consider $n+-n=0$, for some $n \in \mathbf{N}$

$\Longrightarrow \phi(n)+\phi(-n)=0$

$\Longrightarrow \phi(-n)=-\phi(n)$

So, similar arguments can be used for all of $\mathbf{Z}$.

Thus, $\forall n \in \mathbf{Z}, \phi(n)=n$

Lemma 1.3: If $\phi$ is an automorphism $\phi: \mathbf{F} \rightarrow \mathbf{F}$ on an extension field of the rational numbers $\mathbf{Q}(\sqrt{2})$, then the automorphism sends $\mathbf{Q}$ to $\mathbf{Q}$.

Let $p, q, r \in \mathbf{Z}$

Let $s=\frac{p}{q}$ be any rational number.

Need to show that $\phi(s)=s$

$\frac{p}{q}=\frac{p}{q}$

$\Longrightarrow q \frac{p}{q}=p$

So, $\phi(q) \phi\left(\frac{p}{q}\right)=\phi(p)=p$ (By Lemma 1.2 and the fact that $\phi$ is a homomorphism)

$\Longrightarrow q \phi\left(\frac{p}{q}\right)=p$ 
$\Longrightarrow \phi\left(\frac{p}{q}\right)=\frac{p}{q}$

Thus, $\forall q \in \mathbf{Q}, \phi(q)=q$

(Please see this page for the proofs of Lemma 1.2 and 1.3).

\section{$3 \sqrt{2}$ is irrational using Field Automorphisms}

Lemma 1.1: $\sqrt{2}$ is irrational.

Proof:

We know by Lemma 1.2 and Lemma 1.3 that any automorphism from a field extension to itself sends rationals to rationals.

Now consider the automorphism $\phi: \mathbf{Q}(\sqrt{2}) \rightarrow \mathbf{Q}(\sqrt{2})$

Let $\phi(a+b \sqrt{2})=a-b \sqrt{2}$ be any such automorphism for $a, b \in \mathbf{Q}$.

Now, by Lemma $1.3, \forall q \in \mathbf{Q}, \phi(q)=q$

Thus, for the automorphism $\phi, \phi(\sqrt{2})=-\sqrt{2}$

Thus, for this automorphism, $\phi$ does not send $\sqrt{2}$ to itself.

Note that it is sufficient to show this for any one automorphism.

Thus, $\sqrt{2}$ is irrational.

\section{Polynomials}

A polynomial is of the following form:

$f(x)=a_{n} x^{n}+\ldots+a_{1} x+a_{0}$

We can assume that all coefficients $a_{i}$ lie in some field.

The set of numbers that are roots of a polynomial are called Algebraic numbers. 
The set of complex numbers $\mathbf{C}$ is algebraically closed i.e. roots of all possible polynomials lie in the complex field. Every polynomial over $\mathbf{C}$ completely factors or splits over $\mathbf{C}$.

$f(x)=\left(x-r_{1}\right)\left(x-r_{2}\right) \ldots\left(x-r_{n}\right)$

A polynomial is called reducible if it factors or splits over the field. All polynomials split over the field of complex numbers. If the polynomial does not split, it is called irreducible, otherwise reducible.

e.g. $f(x)=x^{2}-x-6$ splits over $\mathbf{Q}$ into $(x+2)(x-3)$. This polynomial is reducible over the field of rational numbers.

\subsection{Eisenstein's Criterion}

A polynomial $f(x)=a_{n} x^{n}+\ldots+a_{1} x+a_{0}$ is irreducible over $\mathbf{Q}$ if the following hold for some prime $p$ :

1. p does not divide $a_{n}$

2. $\mathrm{p}$ divides all $a_{k}$ for $k=0,1 \ldots n-1$

3. $p^{2} \neq a_{0}$

This criterion is not an if and only criterion. A polynomial that satisfies the criterion is irreducible but if it does not satisfy the criterion, it does not mean with certainty that the polynomial is reducible.

\section{$5 \sqrt{2}$ is irrational using Eisenstein's Criterion}

Lemma 1.1: $\sqrt{2}$ is irrational.

Proof:

Consider the polynomial $f(x)=x^{2}-2$. Here $a_{2}=1, a_{0}=2$. The rest of the coefficients are all 0.

For any prime, say 2, all criteria of Eisenstein are met. Thus the polynomial is irreducible over $\mathbf{Q}$.

So, $f(x)=x^{2}-2=0$ does not split into $f(x)=(x-\sqrt{2})(x+\sqrt{2})$ over Q

Thus the polynomial does not have roots in $\mathbf{Q}$. Thus $\sqrt{2} \notin \mathbf{Q}$

Thus $\sqrt{2}$ is irrational. 